\title{
Isolation of Antibiotics Producing Actinobacteria from Biowaste Soil Samples
}

\author{
Rahel Tilahun \\ Department of Biotechnology, School of Biological and Chemical Science, Addis Ababa Science and Technology University, Addis Ababa, \\ Ethiopia
}

Email address:

raheltilahun01@gmail.com

\section{To cite this article:}

Rahel Tilahun. Isolation of Antibiotics Producing Actinobacteria from Biowaste Soil Samples. International Journal of Microbiology and Biotechnology. Vol. 5, No. 4, 2020, pp. 203-206. doi: 10.11648/j.ijmb.20200504.15

Received: November 19, 2020; Accepted: December 4, 2020; Published: December 6, 2020

\begin{abstract}
Actinomycetes are a filamentous bacterium that belongs to the phyla actinobacteria. They are one of the most attractive sources of antibiotics. In this study, Isolation of Actinomycetes strain was obtained by serial dilution method and grown on starch casine agar (SCA). Soil sample were collected from Addis Ababa Science and Technology University (AASTU) and tests were performed to assess the presence of Actinomycetes using macroscopic observation and microscopic methods. Potential actinomycete isolates were also identified as gram positive by using Gram staining technique. The five out of twelve isolates showed coiled and rod shaped spore resembling the genus Streptomycets and antibacterial activities were evaluated for their antibacterial activity. Three out of five potential actinomycete isolates (isolate number 2, 4, and 5) showed anti bacteria activity, of which one isolate (isolate number 5) showed good inhibitory result when tested against two strains of microorganisms (E.coli and S. aures). Isolate 5 was found to exhibit significant antibacterial activity against the gram positive bacteria, Staphylococcus aures. It is concluded that the biowaste soil sampled from AASTU campus harbors a variety of potential antibiotic producing Actinomycetes, which can be used up for further exploration.
\end{abstract}

Keyword: Actinomycetes, Antibiotics, Streptomycets

\section{Introduction}

Actinomycetes are aerobic, free living bacteria, which obtain food by absorbing dissolved organic material, the major source for the production of antibiotics. They are found in soil, freshwater, and marine water. Actinomycetes are a filamentous bacterium that belongs to the phyla actinobacteria and the order actinomycetals; it is also the most invaluable prokaryotes in medical and biotechnology industries due to their ability in producing a vast number of bioactive molecules, mainly antibiotic compounds [1]. They are gram-positive bacteria having high $\mathrm{G}+\mathrm{C}$ content in their DNA and originally considered as an intermediate group between bacteria and fungi.

Actinomycetes are important bacteria due to their ability to produce a wide array of other secondary metabolites, such as antibiotics, antitumor agent, immunosuppressive agent, cosmetics, vitamins, nutritional materials, herbicides, pesticides, anti-parasitic agents and enzymes [2]. Previous studies indicate that soil contains a diverse community of
Actinomycetes with potential activity for isolation of different types of potent antibiotic producing soil actinomycetes [3] Several ecological studies on soil actinomycetes collected from various habitats like grasslands, beach sands, under-ground caves, rice-paddies, orchards and sub-glacial ice of Antarctica, biological wastes, and from soil [4]. Around 23,000 bioactive secondary metabolites produced by microorganisms have been reported and over 10,000 of these compounds are produced by Actinomycetes, representing $45 \%$ of all bioactive microbial metabolites discovered Around 23,000 bioactive secondary metabolites produced by microorganisms have been reported and over 10,000 of these compounds are produced by Actinomycetes, representing $45 \%$ of all bioactive microbial metabolites discovered. Within Actinomycetes, Streptomycetes alone produce 7600 compounds [5]. Many of these secondary metabolites are potent antibiotics which have made streptomycetes the primary antibiotic-producing organisms exploited by the pharmaceutical industry [6]. Hence, the present study is aimed to screen and isolate potent 
antibiotic producing Actinomycetes from the collected soil samples in order to contribute to the problem of antibiotics resistance.

Actinomycetes grow both on the surface of substratum and in to form a substrate branching mycelium and conidia formation, which are similar to those of fungi.

Almost $80 \%$ of the world's antibiotics are known to come from Actinomycetes, mostly from the genera Streptomyces and Micromonospora [2]. At present time most of the diseases caused by bacteria have become resistant to most of the antibiotic [7], which is harmful to the health of the large population of the community. Moreover, it has been a serious problem in the treatment of infectious diseases like pneumonia, diabetes, cancer, vascular disease, and lung disease [8].

Discovery of novel antibiotics from actinomycete is important in helping to crop with that growing proportion of antibiotics resistance bacterial infection that comes untreatable. Hence, this study was conducted with the aim of isolating and screening for antibiotic producing actinomycete from biowaste soil samples.

\section{Material and Method}

\subsection{Soil Sample}

A biowaste soil sample weighing approximately $100 \mathrm{~g}$ was collected from students' cafeteria dumpsite in Addis Ababa Science and Technology University (AASTU). The soil samples were collected from a depth of $15 \mathrm{~cm}$ and were kept in plastic bags. It was then dried in a hot air oven at $60-65^{\circ} \mathrm{C}$ for $1 \mathrm{hr}$ and stored at $4^{\circ} \mathrm{C}$ until use.

\subsection{Culture Media}

Isolation of actinobacteria was carried out using starch casein agar (SCA) according to the method described by [9]. Nutrient agar (Merck, Germany) was used for primary screening of antibiotic producing actinomycetes by spreadplating method with the $\mathrm{pH}$ adjusted to 7 .

\subsection{Test Bacteria}

Gram-positive bacteria (staphylococcus aureus) and Gramnegative bacteria (E. coli) were employed for screening of antibiotic producing actinobacteria isolated from the soil sample. All test bacteria were obtained from microbiology laboratory, AASTU originally from Ethiopian Public Health Institute (EPHI) and handled as per standard procedures.

\subsection{Isolation of Pure Culture of Actinomycetes}

One gram of soil was taken and serially diluted in test tubes containing sterile water up to $10^{-6}$ dilution using standard serial dilution technique. Precisely $100 \mu 1$ of $10^{-1}, 10^{-2}$, $10^{-3}, 10^{-4}$ and $10^{-5}$ dilutions were pipetted and spread over the surface of SCA plates supplemented with Rifampicin $(50 \mathrm{mg} / \mathrm{ml})$ and Flucazol $(33 \mathrm{mgll})$ to inhibit fungal and bacterial growth respectively. All plates were incubated at $28^{\circ} \mathrm{C}$ for 5 days.

\subsection{Morphological and Chemical Identification of Actinobactrial Isolates}

Actinomycete colonies were identified morphologically by using a compound microscope and gram staining technique [10]. The microscopic observation was done at 1000x magnification employing oil immersion. The morphological observation was compared with Bergey's manual of Determinative Bacteriology and the organisms were identified.

Gram staining was done based on the ability of the isolates to retain the purple color or crystal violate during decolonization with alcohol. Gram negative bacteria are decolorized by the alcohol whereas gram positive bacteria were not decolorized after decolonization, safranin, a red counterstain, is used to impart a pink color to the decolorized gram negative organisms.

\subsection{Screening of Antibacterial Activity}

Preliminary screening for antibiotic activity of the isolates was done by using spread-plating technique on nutrient agar medium. Each pure isolate was grown individually on different agar plates. The plates were then incubated at $28^{\circ} \mathrm{C}$ for 5 days followed by overlying of properly diluted test organisms [Gram positive bacteria (Staphylococcus aureus) and Gram negative bacteria $(E$. coli)] to evaluate/ test antibacterial activities of the isolates. Then, the zone of incubation was checked after incubation of the test plates at $37^{\circ} \mathrm{C}$ for $24 \mathrm{hrs}$. In addition, the sensitivity of test organism to selected actinobacterial isolates was tested by agar diffusion assay. Crude extracts of a few screened isolates were prepared and evaluated for antibacterial activities against the test bacterial species. Here, a ditch was made in the agar plate cultured with test organisms using a sterile needle or sterile spatula. The ditch of the culture plate was filled approximately with $200 \mathrm{~mL}$ of crude actinobacterial culture solutions and the plates were incubated at $37^{\circ} \mathrm{C}$ for $24 \mathrm{hrs}$.

\subsection{Data Analysis}

In this study, simple macroscopic and microscopic observations were employed to identify and characterize the actinobacterial isolates. Macroscopic characters include colony characteristics such as size, shape, and color of spore formation. Cultures are observed for microscopic features including aerial mycelium, presence of sclerotia, and spore chain morphology. Formation of inhibition zone against test organisms was used to evaluate antimicrobial activities of actinobacterial isolates by using an agar diffusion method.

\section{Results}

The serially diluted soil samples spread and grown on SCA plates exhibited microbial growth after 5 days of incubation at $28^{\circ} \mathrm{C}$. Approximately 12 bacterial colonies were selected from the original plates of $10^{-1}, 10^{-2}, 10^{-3}, 10^{-4}$ and $10^{-5}$ dilution. The selected isolates colonies were further 
screened using spread plate method and each plate was labeled as $1,2,3,4,5,6,7,8,9,10,11$ and 12 (Figure 1). Actinobacterial growth was observed on each plate and also subcultured onto SCA medium using streak plating technique (Figure 2).

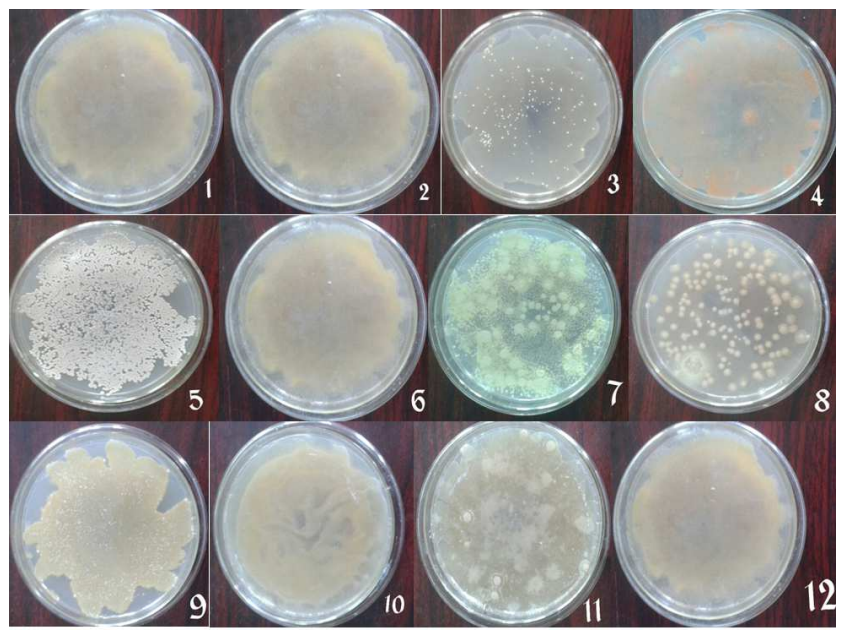

Figure 1. Actinobacterial isolation by using spread plate method.

The actinobacteria isolates were subjected to morphological and gram staining. Table 1 represents the morphological characteristics of the Actinomycetes isolates as studied by colony characterization (color) and Gram staining.

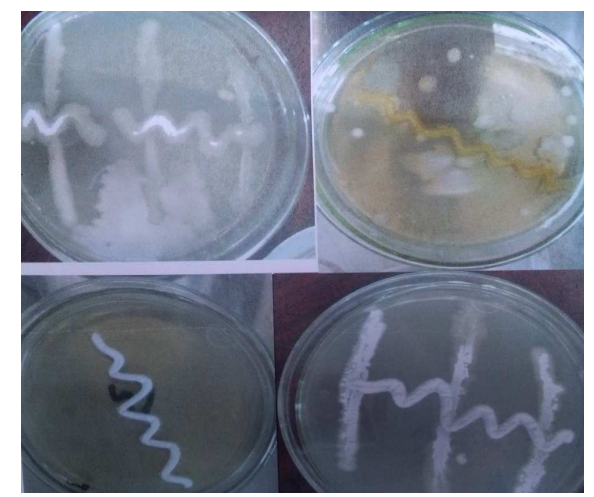

Figure 2. Actinomycetes isolated on SCA medium using streak plating technique.

Table 1. orphological and Chemical characterization of actinobacterial isolates.

\begin{tabular}{llll}
\hline Isolates/plate label & Colony color & Shape & Gram staining \\
\hline 1 & Creamy & Coccus & - \\
2 & Creamy & Bacillus & + \\
3 & Dusty & Rod & + \\
4 & Pink & Rod & + \\
5 & Chalky & Rod & + \\
6 & Creamy & Rod & + \\
7 & Whitish & Bacillus & + \\
8 & Whitish & Coccus & - \\
9 & Dusty & Rod & + \\
10 & Creamy & Bacillus & + \\
11 & Chalky & Rod & - \\
12 & Creamy & Bacillus & + \\
\hline
\end{tabular}

The antibacterial activity of Actinomycetes isolates determined by giant colony technique is shown in Figure 3. In giant colony technique, three isolates showed antibacterial activity. Comparatively, $S$. aures was highly sensitive than other bacteria; $E$. coli showed resistance against the actinomycete tested. The results are tabulated in Table 2.

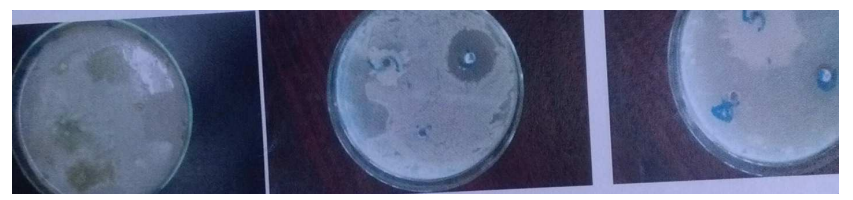

Figure 3. Inhibition activities of Actinomycete isolates against test organisms.

Table 2. Inhibition activities of Actinomycete isolates against test organisms.

\begin{tabular}{llllllllll}
\hline \multirow{2}{*}{$\begin{array}{l}\text { Test } \\
\text { organism }\end{array}$} & \multicolumn{7}{l}{ Isolates of Actinomycetes } \\
\cline { 2 - 10 } & $\mathbf{2}$ & $\mathbf{3}$ & $\mathbf{4}$ & $\mathbf{5}$ & $\mathbf{6}$ & $\mathbf{7}$ & $\mathbf{8}$ & $\mathbf{9}$ \\
\hline E.coli & - & - & - & - & - & - & - & - \\
S.aures & + & - & + & + & - & - & - & - \\
\hline
\end{tabular}

The three isolates of actinomycetes that showed antibacterial activity are 2, 4, 5 in $S$. aures test organism but $E$. coli showed resistance against the isolates, which means they didn't show antibacterial activity. $S$. aures is highly sensitive against 4 and 5 isolates of actinomycetes.

\section{Discussion}

Antibiotics are the most important bioactive compounds for the treatment of infectious diseases. Now, there are basic challenges for effective treatment of infectious diseases because of the emergencies of multi-drug resistance pathogens. Thus, due to the burden for high frequency of multi drug resistant pathogens in the world, there has been an increasing interest for in searching effective antibiotics from soil actinomycetes in diversified ecological niches [11].

In this study, the isolates of Actinomycetes on the plates were identified based on their typical macro and microscopic characters color, dryness, rough, convex colonies they demonstrate. Most of the isolates showed sporulation, with compact chalky, gray, dusty, pink, and creamy like dry colonies with spore formation relevant to that of the species of the genus Streptomyces. They showed pigment-producing ability on SCA is by reason of enough amount of nutrient available in the media [12]. In the current study, the morphological examination of eight out of twelve isolates clearly indicated that they belong to Actinomycetes with particularly closeness to the genera of streptomycetes within streptomycetaceae family (spore chains with coiling and branching features). The reason for such different morphological characters observed could describe that the biowaste soil area harbors diverse potential microorganisms relevant to the group belonging to actinomycetes.

The present study of primary screening antibiotic inhibition experiment indicated that, three $(37.5 \%)$ out of eight potential actinomycete isolates (Isolates 2, 4, and 5) showed possible antimicrobial activity against one test bacteria, the gram positive bacteria, Staphilococus aures by using agar diffusion 
method. This result is not considered much higher or lower figure when compared with similar studies previously reported [13]. In another study, 6 out of 20 strains showed activity against Gram-positive test organisms and only 4 strains showed activity against Gram-negative organisms [14].

Observation of clear inhibition zones around the ditches/ wells on the inoculated plates using agar diffusion method is an indication of antimicrobial activities of antibiotics extracted from actinomycetes against test orgamism [15]. The crude culture solution of the isolates 4 and 5 were tested against $E$. coli and $S$. aures test organisms. The crude culture solution from isolate 5 that showed strong activity against the gram positive $S$. aures but not to the gram negative $E$. coli. The crude culture solution from isolate 5 showed good inhibition zones; like that of the positive control rifampicin. This indicates that further purification of the crud culture from isolate 5 would probably result in stronger inhibition and would be a significant antibiotic substance for the application of treatment of different pathogenic gram positive bacteria.

\section{Conclusion}

The present investigation is aimed to isolate Actinomycetes from the biowaste soil sample in AASTU campus students' cafeteria waste site. Accordingly, actinomycetes were successfully isolated and screened for their antibiotic potential against test pathogenic bacteria. Most interestingly, actinomycete isolate number 5 showed good antibacterial activity against staphylococcus aureus.

Thus, this study has screened antibiotic producing actinomycetes from biowaste soil samples which may give an important clue and lead towards finding novel secondary metabolites (antibiotics) which can be replicated, upscaled, or optimized for treatment of drug-resistant pathogens. The results of the present study may be significant to initiate many researchers and students in this particular area to look for different sources of samples for searching new bioactive secondary metabolites like antibiotics from actinomycete isolates found in diverse environments.

\section{List of Abbreviation}

AASTU: Addis Ababa Science and Technology University SCA: Starch casein

EPHI: Ethiopian Public Health Institute

\section{References}

[1] Goodfellow, M., and Williams, S. T. (1983). Ecology of actinomycetes. Annual review of microbiology. pp. 37 (1): 189-216.

[2] Gouse B. S, Muazzam S. M. Gokul S. S, Ranjith M. S (2017).
Isolation and characterization of actinomycetes from soil of ad-dawadmi, saudi arabia and screening their antibacterial activities. International Journal of Pharmacy and Pharmaceutical Sciences. pp. 9 (10): 975-1491.

[3] Kannan R. R, Vincent S. G (2011). Molecular characterization of antagonistic streptomyces isolated from a mangrove swamp. Asian Journal of Biotechnology. pp. 3 (3): 237-243.

[4] Zanane C, Latrache H, Elfazazi K, Zahir H, and Ellouali M (2018). Isolation of actinomycetes from different soils of Beni Amir Morocco.

[5] Alanis AJ (2005). Resistance to antibiotics: are we in the post-antibiotic era? Archives of Medical Research. pp. 36: 697-705.

[6] Baltz RH (2006). Marcel Faber Roundtable, Is our antibiotic pipeline unproductive because of starvation, constitution or lack of inspiration? Journal of Industrial Microbiology and Biotechnology. pp. 33: 507-513.

[7] Ganesan P, David R. H. A, Reegan A. D (2017). Isolation and molecular characterization of actinomycetes with antimicrobial and mosquito larvicidal properties. Beni-Seuf University Journal of Basic and Applied Sciences. pp. 6 (2): 209-217.

[8] Rotich, M. C. (2018) Bio-Prospecting for Broad Spectrum Antibiotic Producing Actinomycetes Isolated from Virgin Soils in Kericho County (Doctoral dissertation, JKUATCOHES).

[9] Kuster E, Williams ST (1964). Selective media for the isolation of Streptomycetes. Nature, pp. 202: 928-929.

[10] Kawato M, Shinobu R (1979). A simple technique for the microscopical observation. Memoirs of the OsakaUniversity Liberal Arts and Education. pp. 8: 114.

[11] Abo-Shadi M, Sidkey NM, Al-Mutrafy AM (2010). Antimicrobial agent producing microbes from some soils' rhizosphere. The Journal of American Science. pp. 6 (10): 915-925.

[12] Parmar R. S, Singh C, Saini P. and Kumar A. (2016). Isolation and screening of antimicrobial and extracellular pigment producing actinomycetes from Chambal territory of Madya Pradesh region, India. Asian Journal of Pharmaceutical and Clinical Research. pp. 9 (1): 157-160.

[13] Nanjwade B. K, Chandrashekhara S, Shamarez A. M, Goudanavar S. P, Manvi V. F (2010). Isolation and morphological characterization of antibiotic producing actinomycetes. Tropical Journal of Pharmaceutical Research. pp. 9 (3): 231-236.

[14] Wahab A, Shumaila S, Subhan S. A, Ali S. T. and Mujahid T. $Y$ (2015). Isolation and identification of actinomycetes isolated from Karachi soil and screening of antimicrobial compounds. International Journal of Current Research. pp. 7 (2): $12760-12765$.

[15] Okudoh, V. I. (2001). Isolation and identification of antibiotic producing microorganisms from natural habitats in the KwaZulu-Natal midlands (Doctoral dissertation). 